\title{
Emerging role of Natural killer cells in oncolytic virotherapy
}

This article was published in the following Dove Press journal:

ImmunoTargets and Therapy

3I March 2015

Number of times this article has been viewed

\author{
Rauf Bhat \\ Jean Rommelaere \\ Division of Tumor Virology, German \\ Cancer Research Center, Heidelberg, \\ Germany
}

\begin{abstract}
Natural killer (NK) cells constitute a subtype of lymphocytes that initiate innate immune responses against tumors and virus-infected cells. The ability of NK cells to kill target cells or to produce cytokines depends on the balance between signals from activating and inhibitory cell-surface receptors. Therapies with NK cells involve activation of endogenous NK cells and/or exogenous transfer by hematopoietic stem cell transplantation/adoptive cell therapy. To exploit the diverse functional abilities of NK cells for cancer immunotherapy, it is important to understand NK cell biology and the underlying regulatory mechanisms. The state of immune suppression prevalent in malignancies creates the need for innovative therapies. Oncolytic viruses are novel anticancer agents showing selective tropism for tumor cells and lacking pathogenicity in humans, but the use of oncolytic virotherapy (OVT) presents multiple challenges. An increasing body of evidence suggests that the host immune response may critically influence the outcome of OVT. Classically, the immune system is thought to limit the efficacy of therapy through virus clearance mediated by innate immune effectors or through adaptive antiviral immune responses eliminating infected cells. Effective strategies do need to be designed in OVT to circumvent the early antiviral activity of NK cells and to augment late NK-cell-mediated antitumor responses. The intrinsic immunostimulating capacity of oncolytic viruses and the possibility of engineering them to express heterologous immunostimulatory molecules (eg, cytokines) support the use of these agents to enhance antitumor immune responses besides inducing direct oncolytic effects. OVT has indeed shown promising therapeutic outcomes in various clinical trials. Here, we review the biology of NK cells, strategies involving NK cells for achieving cancer therapy, and, more particularly, the emerging role of NK cells in OVT.
\end{abstract}

Keywords: natural killer cells, parvovirus, oncolytic virus, tumors, virotherapy

\section{Introduction Oncolytic virotherapy}

The oncolytic properties of some viruses were first suggested by DePace in 1912 after observing cervical tumor regression associated with rabies virus infection. This paved the way for the first clinical trial of oncolytic virotherapy (OVT) in cancer patients. ${ }^{1}$ The past few decades have seen a revival of the concept of using viruses as therapeutic agents against cancer because, despite constant advances cancer therapy, conventional treatments by surgery, chemotherapy, or radiotherapy remain partly ineffective. This revival is reflected in the fact that oncolytic viruses (OVs) (eg, herpes simplex virus, vaccinia virus, reovirus, and adenoviruses) are now in Phase III clinical trials, with encouraging results confirming the potential of this therapeutic strategy. Besides displaying good safety profiles in humans, OVs must show antitumor efficacy. Intense
Correspondence: Rauf Bhat

Deutsches Krebsforschungszentrum,

Tumor Virology, FO I0, Im Neuenheimer

Feld 242, D-69120, Heidelberg, Germany

Tel $+49622 \quad 1424961$

Fax +49 622 I 424962

Email rauf0I2@yahoo.com 
efforts are thus needed to improve their reactivity, notably by incorporating therapeutic genes into the viral genomes, facilitating virus biodistribution and tipping the immune balance in favor of antitumor (as opposed to antiviral) effects. It is further anticipated that greater anticancer effectiveness may be achieved through combination therapy including OVT. Therefore, considerable efforts have also been invested in evaluating the combination of OVT with radio-, chemo-, and immunotherapies. ${ }^{2}$

OVs are self-replicating and able to lyse tumor cells selectively while sparing normal cells. They demonstrate a natural preferential tropism for tumor cells and can be genetically modified to show enhanced oncotropism. The advantage is that tumor cells show impaired antiviral responses, including a deficient interferon (IFN) response, and higher permissiveness toward viral replication. To be rendered dependent on these features of tumor cells, some OVs (eg, adeno, measles, herpes, polio, and vaccinia viruses) must be engineered by modifying or deleting specific viral genes. ${ }^{3}$ Importantly, besides killing tumor cells directly, OVs have the capacity to stimulate the anticancer immune response. OV oncosuppression thus includes at least two major arms: virus-induced oncolysis and virus-mediated immunostimulation. It follows that the immune system acts as a two-edged sword in OVT, interfering both negatively with virus propagation and positively with anticancer immunity. It is thus essential to gain greater insight into the roles of the immune system in virotherapies. To enhance the oncosuppressive action of OVs, transgenes encoding immunostimulating cytokines (eg, granulocyte macrophage-colony stimulating factor [GM-CSF], interleukin [IL]-2, etc) have been incorporated into viral genomes to induce local and systemic immune responses.

A promising candidate for OVT is the rodent protoparvovirus, briefly discussed in the next section to illustrate the many-faceted aspects of this therapeutic modality, with emphasis on the involvement of the immune system in OVmediated oncosuppression.

\section{Rodent protoparvovirus: promising OVs}

Members of the rodent protoparvovirus species (PV) are promising candidate oncotherapeutic agents because of their natural oncotropism, because humans have no pre-existing immunity against them and because they lack pathogenicity in humans. PVs belong to the Parvoviridae family and are small nonenveloped icosahedral particles (around $25 \mathrm{~nm}$ in diameter) containing a single-stranded DNA genome about 5000 nucleotides long. Two PVs have been particularly well characterized: the minute virus of mice (MVM) and H-1PV, whose natural hosts are mice and rats, respectively. The PV genome consists of two transcriptional units, one encoding nonstructural proteins (NS1/2) and the other encoding capsid proteins (VP1/2). Expression of the NS1/ NS2-encoding genes is controlled by the early $\mathrm{P} 4$ promoter, while VP1/VP2 gene expression is controlled by the late p38 promoter, itself induced by NS1. The major nonstructural protein NS1 is essential to both virus replication and viral cytopathic effects in transformed cells. The oncosuppressive action of PVs has been demonstrated in both in vitro systems and animal models. PV oncoselectivity is due in part to the strict dependence of the onset of the PV lifecycle on S-phase-associated cellular replication and transcription factors. PV oncotropism is further favored by various cellular factors that are modulated by malignant transformation and play a positive role in virus replication, survival, and cytopathic effects. ${ }^{4,5}$ The intracellular environment promoting the growth of tumor cells thus appears also to boost the PV lifecycle. In addition, the fact that transformed mouse cells are deficient in the ability to evade the virus-induced type I IFN-mediated antiviral response in transformed mouse cells may contribute to their targeting by parvoviruses. H-1PV, however, does not appear to rely on this deficiency for selective human tumor cell targeting. ${ }^{6}$ Under in vivo conditions, PV-mediated killing of tumor cells cannot be attributed solely to the oncolytic properties of the viruses. By lysing tumor cells, OVs induce the production and release of both tumor-associated antigens (TAAs) and immunostimulating factors. The capacity of PV-induced oncolysis to activate Natural killer (NK) and dendritic cells (DCs) has been demonstrated in coculture systems. Furthermore, evidence of the contribution of the immune system to PV oncosuppression has been obtained through adoptive transfer, immunodepletion, and immunereconstitution experiments. ${ }^{7}$ Because of the multifaceted beneficial effects of PVs in model systems, H-1PV is currently being tested in patients with recurrent glioblastoma multiforme in a Phase (I/IIa) clinical trial. ${ }^{8}$

\section{OVT and the immune system}

While most attention was initially paid to the direct oncolytic effects of OVs, there is now growing interest in the interactions of OVs with the immune system. The immune system does not distinguish therapeutic from pathogenic viruses and thus challenges OVs by attempting to clear them out of the organism. The defensive role of the adaptive immune response is usually triggered by circulating virion-associated antigens 
or cell-associated viral gene products whose synthesis takes place upon viral infection of target cells. These antigens can be recognized by specific immunoglobulin (Ig) surface receptors on B cells, leading to the activation of these cells and production of neutralizing antiviral antibodies. This raises a general concern that the efficacy of OVs in the diseased host will be limited by the immune system itself. Furthermore, infected tumor cells activate antiviral immunity, inducing antiviral cytokines and the antigen-independent or dependent lysis of virus-infected cells by NK and CD8 ${ }^{+} \mathrm{T}$ cells, respectively. Viral infection can also facilitate complementmediated lysis of infected cells. Depending on the kinetics, these responses can interfere with virus expansion and hamper the activity of OVs. Yet, immune reactions triggered by infected cells can also favor OVT. Several OVs cause infected tumor cells to expose pathogen- and danger-associated molecular patterns to antigen-presenting DCs. Activated DCs produce cytokines that stimulate the adaptive and innate arms of the host's immune system and cross-present TAAs, triggering adaptive cellular immune responses, as evidenced by animal and patient studies. ${ }^{9,10}$ OVs have proven effective in the presence of a functional immune system, and several reports document the existence of a synergistic relationship between direct viral oncolysis and activation of antitumor immune responses. To suppress the host's antiviral immune response and boost antitumor immunity, OVs have been combined with pharmacological adjuvants and armed with therapeutic transgenes. The balance between antiviral and antitumor immune priming in OVT is likely to depend on the route of administration, with intravenous administration tending to favor a virus-specific immune response and local treatments favoring a cellular response. Strategies can thus be devised to exploit the potential of OVs to trigger an antitumor immune response while minimizing the restriction of OV-induced oncolysis by the immune system. This dual impact of the immune system on OVT notably involves NK cells, the main effectors of innate immunity. ${ }^{11}$ This review focuses on the role of NK cells in OVT, after a brief overview of NK cell biology.

\section{NK cell biology Characteristics of NK cells}

NK cells are the third major lymphocyte population after $\mathrm{T}$ and $\mathrm{B}$ cells. The function of NK cells is to recognize and kill a broad range of target cells, including malignant and virusinfected cells. Genetic experiments on mice have linked the spontaneous development of tumors to the absence of NK cells. ${ }^{12}$ The discovery of NK cells dates back to 1975 , when background or natural cytotoxicity was observed in assays measuring ${ }^{51} \mathrm{Cr}$ cytotoxicity against syngenic and allogenic tumors, even when effector cells from athymic mice were used. Cytotoxicity was attributed to cells distinct from T cells, requiring no prior sensitization in order to lyse a target cell in a non-MHC-restricted manner. ${ }^{13,14}$ In addition to their potent cytotolytic activity, NK cells were found to produce a host of immunoregulatory cytokines and chemokines, including IFN - upon encountering a virus - or pathogen-infected cells. NK cell function is regulated by cytokines, particularly IL-2, IL-15, and IL-18, produced, respectively, by activated T cells, macrophages, and dendritic cells. In response to infection, NK cells migrate to inflamed tissues and secondary lymphoid organs to defend against pathogens and tumors. In humans, the absence of NK cells causes clinical symptoms similar to those of classic severe combined immunodeficiency (SCID) syndrome. $^{12}$

Human NK cells are identified by expression of the CD56 molecule and the absence of the T-cell markers CD3 and TCR. On the basis of the level of CD56 expression, NK cells can be further classified as CD56-bright or CD56-dim. The CD56-dim subset comprises the majority of circulating NK cells in the blood, characterized by high cytotoxicity, low cytokine production, and expression of killer immunoglobulin-like receptors (KIRs) in addition to the Fc $\gamma$ receptor CD16. The CD56-bright subset is mostly found in lymph node tissues, and shows low cytotoxicity and produces high amounts of cytokines, including IFN- $\gamma$ and chemokines. CD56-bright cells express neither CD16 nor KIRs, which probably explains their poor killing ability. This subset plays a critical role in modulating the adaptive immune response by regulating DCs and T-cell priming. ${ }^{15}$

\section{NK cell development}

NK cells, like other hematopoietic cells, develop from pluripotent hematopoietic stem cells in the bone marrow, via a common lymphoid progenitor and through a series of differentiation and maturation steps. NK cell progenitors express FcR receptor III and give rise to NK cells upon intravenous transfer. During their development, NK cells start expressing characteristic surface receptors, including CD56 and KIRs (in humans) or NK1.1 and Ly49 (in mice). After acquisition of the IL-2/IL-15R $\beta$-chain (CD122), NK cell precursors become responsive to IL-15, a cytokine essential to the maturation and development of NK cells. NK cells also undergo differentiation in peripheral tissues, including both lymphoid and non-lymphoid organs..$^{15,16}$ 


\section{NK cell activating and inhibitory receptors}

The finely tuned function of NK cells is controlled by a complex repertoire of activating and inhibitory signals generated by a multitude of receptors triggering cytotoxicity and the release of chemokines and cytokines. The activity of NK cells must be regulated in order to prevent them from attacking normal healthy cells. NK cells express an array of inhibitory receptors recognizing self-HLA class I molecules. Normal and healthy cells express sufficient levels of HLA class I ligands, which enables them to resist NK-cell-mediated killing. In contrast, tumor cells and virus-infected host cells undergo downregulation of HLA-Class I ligands, sensitizing them to NK cell attack. In addition to a lack of inhibitory ligands, tumor cells and virus-infected cells also express selfinduced, altered-self, or pathogen-encoded molecules, which bind to the corresponding activating receptors on NK cells, triggering NK cell cytotoxicity. ${ }^{17} \mathrm{NK}$ cells express activating or inhibitory receptors in diverse combinations, and this gives rise to functionally distinct subsets of NK cells. In humans, several KIRs triggering NK cell activation, inhibition, or both have been identified (see Table 1). KIRs play a vital role in the development and regulation of NK cells. KIRs possess two or three extracellular Ig-like domains involved in ligand binding and either a long or a short cytoplasmic tail involved in signaling. Inhibitory KIRs bind to distinct HLA class I allotypes and to the ligands of most activating KIRs. KIRs are encoded by the multigenic and polymorphic leukocyte receptor complex and thus display high diversity in individuals and populations, which depends on both allele variability and haplotype differences in KIR gene content. Together with their HLA Class I ligands, KIRs generate a unique range of combinations that determine an individual's health and disease-resistance status. ${ }^{18,19}$

\section{NK cell education}

To safeguard against chronic immune activation and the development of autoimmunity, NK cells need to be educated. Initially, two models were put forward to explain NK cell education. In the "arming" or "licensing" model, NK cells are assumed to be inactive by default and acquire their full functionality (licensing) only through the engagement of an inhibitory receptor. As a consequence of this licensing process, the inhibitory KIR repertoire becomes greatly influenced by self-HLA class I alleles. NK cells expressing KIRs for non-self HLA class I alleles would be "unlicensed" and substantially hyporesponsive.

In the "disarming" model, NK cells are active by default but are rendered hyporesponsive through continuous stimulation via activating receptors recognizing endogenous ligands,
Table I NK cell activatory and inhibitory receptors

\begin{tabular}{|c|c|c|c|}
\hline Receptor type & Ligands & $\begin{array}{l}\text { Signal } \\
\text { type }\end{array}$ & Species \\
\hline KIRs & HLA-A-C & & Human \\
\hline KIR2DLI & HLA-C2 & Inhibitory & \\
\hline KIR2DL2/3 & HLA-CI & Inhibitory & \\
\hline KIR2DL4 & HLA-G & Activatory & \\
\hline KIR2DL5 & Unknown & Inhibitory & \\
\hline KIR3DLI & HLA-Bw4 & Inhibitory & \\
\hline KIR3DL2 & HLA-A3, -AII & Inhibitory & \\
\hline KIR2DSI & HLA-C2 & Activatory & \\
\hline KIR2DS2 & HLA-CI & Activatory & \\
\hline KIR2DS3 & Potentially HLA-C & Activatory & \\
\hline KIR2DS4 & HLA-Cw4 and HLA-II & Activatory & \\
\hline KIR2DS5 & Unknown & Activatory & \\
\hline KIR3DSI & HLA-Bw4 & Activatory & \\
\hline Ly49 & MHC class I & & Mouse \\
\hline Ly49A & $H-2 D^{d, k, p}$ & Inhibitory & \\
\hline Ly49C & $H-2 K^{b, d}, H-2 D^{b, d, k}$ & Inhibitory & \\
\hline Ly49D & $\mathrm{H}-2 \mathrm{D}^{d}$ & Activatory & \\
\hline $\mathrm{Ly} 49 \mathrm{H}$ & $\mathrm{ml} 57$ & Activatory & \\
\hline Ly49I & $\mathrm{H}-2 \mathrm{~K} / \mathrm{D}^{\mathrm{b}, \mathrm{d}, \mathrm{s}, \mathrm{q}, \mathrm{v}}$ & Inhibitory & \\
\hline Ly49P & $H-2 D^{d}$ & Inhibitory & \\
\hline \multirow[t]{2}{*}{ CD94-NKG2 } & Human: HLA-E & & Human/ \\
\hline & Mouse: Qa lb & & mouse \\
\hline NKG2A & & Inhibitory & \\
\hline NKG2C & & Activatory & \\
\hline NKG2E & & Activatory & \\
\hline NKG2D & Human: MIC-A/-B, & Activatory & Human/ \\
\hline & ULBPI/2/3/4, RAE-I, & & mouse \\
\hline & MULT I, H60 & & \\
\hline NCRs & Viral hemagglutinin & & $\begin{array}{l}\text { Human/ } \\
\text { mouse }\end{array}$ \\
\hline NKp30 & BAT-3, HSPG, B7-H6 & Activatory & \\
\hline NKp44 & Viral hemagglutinin & Activatory & \\
\hline NKp46 & Viral hemagglutinin, HSPG & Activatory & \\
\hline NKp80 & $\mathrm{AICL}$ & Activatory & \\
\hline $\begin{array}{l}\text { LILR (ILT, LIR, } \\
\text { CD85) }\end{array}$ & MHC class I, ULI8 & Inhibitory & $\begin{array}{l}\text { Human/ } \\
\text { mouse }\end{array}$ \\
\hline 2B4 (CD244) & CD48 & $\begin{array}{l}\text { Activatory/ } \\
\text { inhibitory }\end{array}$ & $\begin{array}{l}\text { Human/ } \\
\text { mouse }\end{array}$ \\
\hline KLRGI & Cadherins & Inhibitory & $\begin{array}{l}\text { Human/ } \\
\text { mouse }\end{array}$ \\
\hline NKR-PI & Ocil/Clr-b & $\begin{array}{l}\text { Activatory/ } \\
\text { inhibitory }\end{array}$ & Mouse \\
\hline PILR & CD99 & Activatory & Mouse \\
\hline \multirow[t]{2}{*}{ DNAM-I } & PVR & Activatory & Human/ \\
\hline & CDI22 & & mouse \\
\hline CEACAMI & CEACAMI & Inhibitory & Human/ \\
\hline (CD66a or BGP) & CEACAM5 & & mouse \\
\hline SIGLEC7/9 & Sialic acid & Inhibitory & Human \\
\hline SIGLEC-E & Sialic acid & Inhibitory & Mouse \\
\hline
\end{tabular}

Abbreviations: KIR, killer immunoglobulin-like receptor; RAE-I, retinoic acid early transcript-I; MULT I, mouse ULI6-binding-like transcript-I; NCR, natural cytotoxicity receptor; BAT-3, HLA-B-associated transcript 3; LILR, leukocyte immunoglobulin-like receptor; KLRGI, killer cell lectin-like receptor GI; CEACAM, carcinoembryonic antigen-related cell-adhesion molecule; PVR, polio virus receptor; NK, natural killer.

unless counteracted by signals of inhibitory receptors. Though inhibitory receptors have opposite roles in both models, the outcome is similar - only NK cells with an inhibitory receptor for self-MHC class I can become functionally active. The education of NK cells is not simply an on/off process, but 
can be quantitatively tuned. Various studies have suggested that the NK cell education process is reversible. This explains why another model, called the "rheostat" model, has been proposed. This model supplements the arming and disarming models and describes NK cell education as a dynamic process. For example, NK cells lacking sufficient inhibitory receptors for self-MHC class I are not deleted but rendered hyporesponsive. Under certain circumstances, such as during an acute virus infection, these hyporesponsive cells can become functional under the influence of proinflammatory cytokines secreted by immune cells..$^{20,21}$

\section{NK cell applications in cancer immunotherapy}

The inherent ability of NK cells to recognize and kill tumor cells makes them promising candidates for cancer immunotherapy. NK cells act in a non-antigen-specific manner and bind to a wide panel of ligands through their broad repertoire of activating and inhibitory receptors. This enables them to target various types of tumors. NK cells are convenient to isolate, stimulate, and expand ex vivo for subsequent use in adoptive or autologous transfer therapy. Consequently, NK cells represent a potential alternative or adjuvant to conventional therapies. ${ }^{22}$

\section{Cytokine modulators of endogenous NK cell activity}

Various modulators have been employed to enhance the ability of NK cells to kill tumor cells. IL-2 and IL-15 are of special interest, as they play an essential role in NK cell survival, proliferation, and functioning. IL-2 has been widely used for ex vivo activation of NK cells to lymphokineactivated killer cells. Clinically, IL-2 is used against renal cell carcinoma and melanoma, with encouraging responses, but repeated high doses of IL-2 cause morbidities such as vascular leak syndrome due to the selective expansion of the CD5-bright subset and of regulatory T cells (Tregs) competing with NK cells for IL-2. In addition, transforming growth factor (TGF)- $\beta$ and IL-10 produced by Tregs inhibit NK cell functions. Therefore, Treg depletion before administration of IL-2 has been recommended. ${ }^{23}$ Another cytokine of interest, IL-15, is produced by activated macrophages and dendritic cells. Unlike IL-2, IL-15 does not stimulate Tregs but induces activation and expansion of memory CD8 T cells and both subsets of NK cells. Moreover, IL-15 has proven less toxic than IL-2 in primate models. ${ }^{24}$

Among the other immunomodulators of NK cells, bortezomib, a protease inhibitor causing the upregulation of the death receptors Fas and DR5, cooperates with NK cells in initiating an apoptotic cascade in tumor cells. In addition, bortezomib decreases the amount of MHC-I molecules on the surfaces of tumor cells, thereby enhancing their susceptibility to NK cells. The toxicity of bortezomib, however, limits its application in NK cell therapy. ${ }^{25}$ Another approach to increasing NK-cell-mediated killing of tumor cells involves the use of an antibody cross-reacting with KIR2DL receptors and thus preventing their inhibitory signaling. Administration of this antibody was found to boost NK induced killing of autologous and HLA-matched leukemia cells. ${ }^{26}$

\section{NK cell transplantation}

Hematopoietic stem cell transplantation (HSCT) with bonemarrow- or blood-derived stem cells is increasingly used to treat hematological malignancies and nonmalignant disorders. More potent graft-versus-tumor effects are achieved with haploidentical transplants from half-matched donors (family members with one identical and one mismatched HLA haplotype as compared to the recipient). Following allogenic HSCT, NK cells are among the first to undergo maturation and to recover, and are believed to play an important role in preventing tumor recurrence in the recipient patient. NK receptors for MHC Class I molecules (notably KIRs) are major determinants of this anticancer response. In particular, donor NK cells expressing KIRs that are not engaged by any of the HLA Class I alleles expressed by the recipient (due to KIR-ligand mismatch) recognize recipient cells as foreign and become effective against them. Because of the loss of HLA molecules and other cell surface alterations accompanying malignant transformation, recipient tumor cells represent privileged targets for alloreactive NK cell attack. ${ }^{27}$

Haplo-HSCT appears a very promising approach to treating children with high-risk leukemias. After haplo-HSCT, however, the NK cells that proliferate initially belong to the poorly cytotoxic, relatively immature CD56-bright subset expressing the HLA-I-specific receptor CD94/NKG2A. It takes a further 4-6 weeks for $\mathrm{KIR}^{+} \mathrm{NK}$ cells to appear, and meanwhile the leukemia may relapse. To shorten this lag, donor-derived mature alloreactive NK cells are infused at transplantation after ex vivo activation with IL-2. However, IL-2-driven expansion of regulatory $T$ cells and the reduced longevity of activated NK cells limit the efficacy of this treatment. ${ }^{27,28}$ Another risk of hematopoietic cell transplantation is the occurrence of graft-versus-host disease. To reduce this risk, $\mathrm{T}$ - and $\mathrm{B}$-cell-depleted transplants have been successfully used. This approach also increases the number of mature NK cells that can promptly exert antileukemia cytotoxicity. ${ }^{29}$ Another approach under current clinical evaluation involves infusion of continuously growing NK cells. The most studied line, NK-92, was derived from 
an NK cell lymphoma and exhibits a CD56-bright/CD16/ $\mathrm{KIR}^{-}$phenotype. As these cells become more cytotoxic upon stimulation with IL-2, a variant called NK-92MI, producing IL-2 via an autocrine mechanism, has been produced. ${ }^{30}$

\section{Role of NK cells in antibody-dependent cell-mediated cytotoxicity}

Targeting tumor cells with antibodies has yielded promising results. One of the mechanisms involved, antibody-dependent cell-mediated cytotoxicity (ADCC), results in lysis of antibody-coated tumor targets by NK cells. ADCC occurs after engagement of CD16 receptors on NK cells by the Fc portion of cell-bound antibodies, triggering tumor cell lysis. Antibodies targeting cancer cells can thus be used to direct NK-cell-dependent ADCC toward tumors. Examples of this strategy include the use of the antibodies rituximab and trastuzumab to target $\mathrm{CD} 20^{+}$lymphomas and HER2 ${ }^{+}$breast tumors, respectively. The anticancer efficacy of these treatments can be further increased by combination with IL-2. Conjugation of both IL-2 and IL-12 to an anti-CD30 antibody for Hodgkin's lymphoma has shown preclinical efficacy in mouse models. ${ }^{31,32}$

\section{NK-cell-based therapy of solid tumors}

In addition to killing a wide variety of tumor cells, NK cells can also target cancer stem cells (CSCs), a small subpopulation of quiescent, self-renewing, chemo- and radio-resistant cells residing within the tumor mass. Several studies have shown a link between the presence of a large number of tumor-infiltrating NK cells and improved prognosis for solid tumors. Yet, the tumor type, the chemokine composition of the tumor microenvironment, and poor trafficking to tumor sites limit the benefits of endogenous or adoptively transferred NK cells. Immunosuppressive cytokines released by tumor or stromal cells, including TGF- $\beta$, macrophage migration-inhibitory factor (MIF), and PGE-2, downregulate the expression of activating NK cell receptors. Furthermore, chronic ligand-induced stimulation and hypoxic conditions can inhibit NK cell activity. ${ }^{33,34}$ Therefore, new strategies are worth devising to counteract tumor escape and to improve the efficacy of NK-cell-based adoptive immunotherapy of solid tumors.

\section{Role of NK cells in oncolytic virotherapy Antiviral role of NK cells in OVT}

NK cells are involved in virus-triggered inflammation through targeting and destruction of virus-infected host cells and production of IFN- $\gamma$. Consequently, they may adversely affect OVT by preventing the intratumoral spread of OVs and thereby limiting the extent of virus-mediated oncolysis. One study, for instance, showed enhanced replication of oncolytic vesicular stomatitis virus (oVSV) and more effective tumor-cell killing after NK cell depletion. ${ }^{35}$ In another work, the antiviral effect of NK cells was circumvented by using a recombinant UL141-encoding virus (rVSV-UL141) blocking CD155 expression on infected cells, thereby diminishing DNAM-1-mediated signaling in NK cells. They found this approach to impede NK cell activation and recruitment and to improve virus spread, causing increased necrosis and enhanced survival of the animals tested. ${ }^{36}$ In the framework of OVT of glioblastoma with oncolytic herpes simplex virus (oHSV), NK cells were shown to preferentially kill oHSVinfected cells, this antiviral effect being due to enhanced activation of NK cells via specific natural cytotoxicity receptors (NCRs) whose ligands were upregulated on the virus-infected target cells. ${ }^{37}$ In other cases, NK cells did not appear to have a major impact on the outcome of OVT. For instance, a study using immunosuppressed nude $\left(\mathrm{MHCII}^{+/-}\right.$cell-depleted) mice and immunodeficient (T/B/NK-cell-deficient) mouse strains showed no significant effect of activated NK cells on vaccinia-virus-mediated tumor regression in a human breast tumor model..$^{38}$ Likewise, human PBMC reconstitution in SCID mice had little effect on the capacity of $\mathrm{H}-1$ parvovirus to suppress the growth of human nasopharyngeal carcinoma xenotransplants. ${ }^{39}$

\section{Supportive role of NK cells in OVT}

Besides the abovementioned negative effect on OV spread, NK (and some other immune) cells have been found, on the contrary, to support OV-mediated tumor clearance in various instances. Evidence of NK cell activation by OV-infected tumors and of participation of these cells in OVT has been obtained for several OVs as discussed here.

i. Herpes simplex virus (HSV). In a metastatic melanoma model, intratumoral therapy with the replication-restricted oncolytic HSV-1716 mutant was found to induce recruitment of $\mathrm{T}$ and NK cells into neoplastic lesions, and was much hampered by T-cell depletion and NK cell deficiency, as attested by poor survival of beige mice. ${ }^{40}$ In an ovarian cancer model, oncosuppression by oHSV was accompanied by increased recruitment of immune cells and upregulation of IFN- $\gamma$, MIG, and IP- $10 .{ }^{41}$ In a breast cancer model, an increased number of NK cells and a mild decrease in Tregs was observed in the spleen upon administration of a novel oHSV type $2 .^{42}$ 
ii. Reovirus. A similar correlation between OVT and immune cells, including NK cells, was observed in a Phase I clinical trial using an intravenously administered reovirus: in this study, a significant increase in circulating CD8 and NK cells was observed. ${ }^{43}$ In support of a positive contribution of both NK cells and CD8 T cells to OVT, these cells were found to mediate, to a large extent, the oncosuppressive activity of an oncolytic reovirus in a prostate cancer model and that of parapoxvirus ovis in multiple cancer models. ${ }^{44,45}$

iii. Coxsackievirus (CV). Furthermore, in a quest for novel oncolytic agents, intratumoral CVB3 administration was found to lead to striking recruitment of NK cells and granulocytes, both of which contributed to antitumor effects against lung adenocarcinoma xenografts. ${ }^{46}$

iv. Measles virus (MV). Recombinant oMV strains retargeted to gliomas by the display of a single-chain antibody against EGFRvIII caused increased intratumoral infiltration of NK cells and macrophages, associated with prolonged animal survival. ${ }^{47}$

v. H-1 parvovirus (H-1PV). The above observations raise the question of how the interplay between OVs, tumors, and NK cells can lead to mobilization of NK cells against neoplastic cells. A clue lies in our finding that, besides having intrinsic oncolytic activity, parvovirus $\mathrm{H}-1 \mathrm{PV}$ renders infected pancreatic ductal adenocarcinoma (PDAC) cells capable of activating co-cultured NK cells in vitro, causing enhanced NKcell-mediated cancer cell killing and production of IFN- $\gamma$, TNF- $\alpha$, and MIP- $1 \alpha / \beta$. Sensitization of H-1PVinfected PDAC cells to NK-cell-dependent killing was attributed to significant downregulation of MHC class I expression and upregulation of the DNAM1 ligand CD155. Our data suggest that NK-cell-mediated innate immune responses against PDAC may be boosted as a result of $\mathrm{H}-1 \mathrm{PV}$-based $\mathrm{OVT}{ }^{48}{ }^{4}$ Similar observations have been made on colon carcinoma cells, where infection with H-1PV resulted in enhanced killing by cocultured NK cells. In this model, NK-cell-dependent killing of cancer cells was found to be mostly mediated by a combination of NCRs, namely NKp30, NKp44, and NKp46, via upregulation of cellular NCR-ligand expression. ${ }^{49}$ The results are illustrated in Figure 1 and indicate that, upon infecting cancer cells, PVs can indirectly activate effector NK cells to infected target cells and, at least to some extent, also uninfected bystander tumor cells. In agreement with this, robust antitumor responses were observed in xenograft models of human PDAC. Treatment with parvoviruses armed with IL-2 or the chemokine MCP-3/CCL7 led to intratumoral recruitment and activation of $\mathrm{NK}$ cells and monocytes. ${ }^{50}$
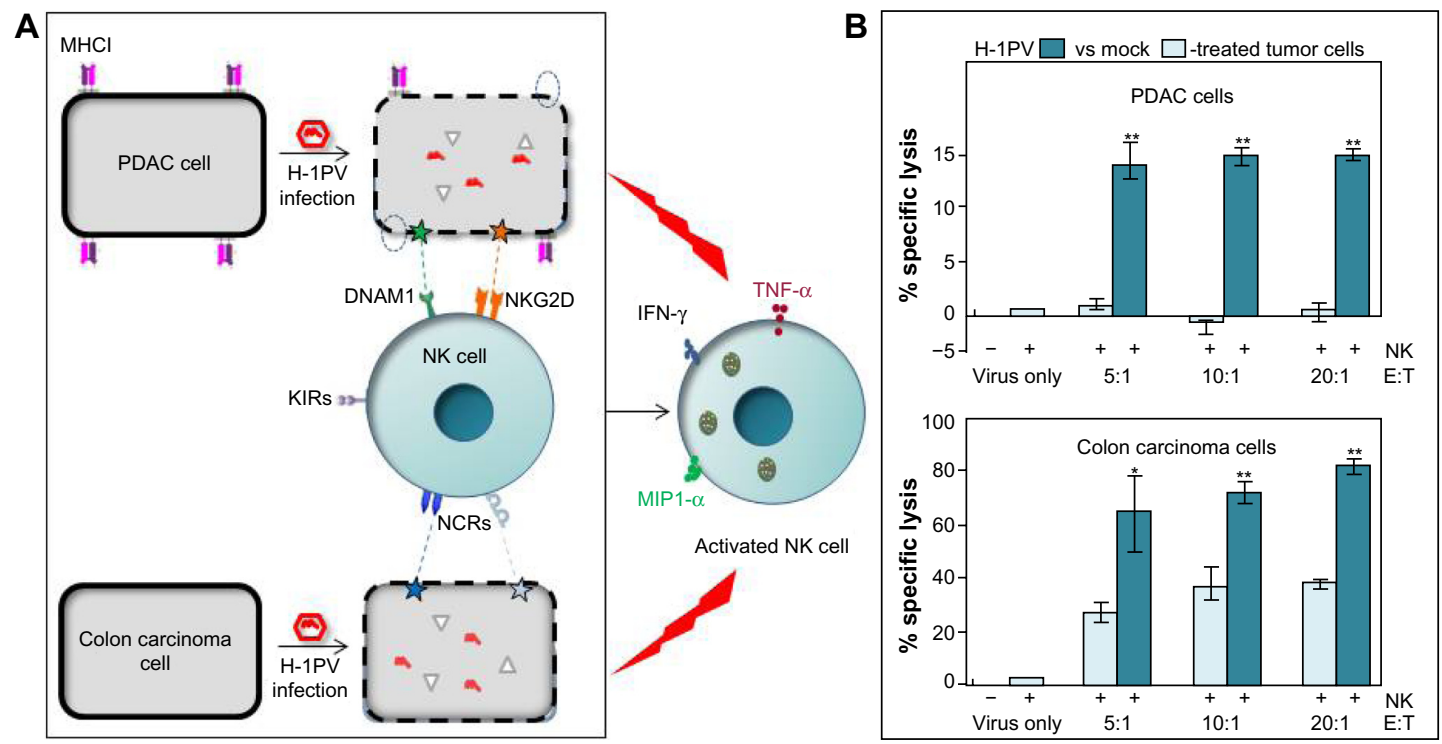

Figure I Schematic presentation of the indirect immunostimulating effects of protoparvovirus $\mathrm{H}$-IPV on human NK cells.

Notes: (A) H-IPV-infected pancreatic ductal adenocarcinoma (PDAC) and colon carcinoma cells were cocultivated with IL-2-stimulated NK cells. Contact with virusinfected tumor cells triggered NK cell activation, resulting in the production of cytokines and chemokines, including IFN- $\gamma$, TNF- $\alpha$, and MIPI- $\alpha$. This activation was traced back to the downregulation of $\mathrm{MHCl}$ (dotted circles) and an upregulation of NK-cell-activating ligands (stars). (B) Activated NK cells (E) showed a strikingly enhanced capacity for killing cocultured PDAC and colon carcinoma target cells $(\mathrm{T})$. $* P<0.05 ; * * P<0.01$.

Abbreviations: $\mathrm{H}$-IPV, H-I parvovirus; IL, interleukin; IFN, interferon; TNF, tumor necrosis factor; NK, natural killer; MIPI- $\alpha$, macrophage inflammatory protein I alpha; KIRs, killer inhibitory receptors; NCRs, natural cytotoxicity receptors; DNAM-I, DNAX accessory molecule-I; NKG2D, natural-killer group 2, member D. 
vi. Myxoma virus (MYXV). In agreement with our findings with the parvovirus human-H-1PV in PDAC and colon carcinoma models, MYXV infection of human glioma cells was found to promote NK-cell-mediated recognition and killing of these cells due to their downregulated surface expression of MHC I. In an in vivo model, NK-cell-mediated lysis of glioma cells improved animal survival, demonstrating the cooperation between MYXV and NK cells in glioma therapy. ${ }^{51}$

vii. Newcastle disease virus (NDV). While cellular cognate ligands of activating NK cell receptors are thought to be induced in tumor cells by oncolytic parvoviruses, viral proteins produced by some other OVs appear to act as direct ligands of these receptors. This is the case of the avian NDV, which stimulates NK cells to produce IFN- $\gamma$ and TNF- $\alpha$ and to lyse NDV-infected tumor cells. The mechanism underlying this activation of NK cells involves expression of viral hemagglutinin neuraminidase (HA) on tumor cells and its binding to the NCRs NKp44 and NKp46 on NK cells. ${ }^{52}$

viii. Influenza virus (IAV). Similarly, infection of prostate cancer cells by a genetically modified IAV (DeltaNS1) was shown to prime NK cells through expression of viral HA on target cells, resulting in enhanced extracellular-signal-regulated kinase phosphorylation and NKp46-mediated granule release by NK cells. ${ }^{53}$

ix. Vesicular stomatitis virus (VSV). Tumor cell changes responsible for NK cell activation can also be elicited by OVs in an indirect way. It has been shown that oVSV induces innate immune sensing cells to secrete type III IFN IL-28 into the tumor microenvironment, causing responsive mouse melanoma cells to display NK cell ligands and become targets for NK cell recognition, activation, and cytotoxicity. ${ }^{54}$ Treatment with another recombinant oVSV expressing tumor suppressor P53 was found to trigger $\mathrm{CD}_{4} 9 \mathrm{~b}^{+} \mathrm{NK}$ - and tumor-specific $\mathrm{CD}^{+} \mathrm{T}$-cell responses in immunocompetent mice with metastatic mammary adenocarcinoma, causing enhanced animal survival. ${ }^{55}$ Moreover, in a B16 melanoma model, NK and T cell depletion decreased the efficacy of VSV-based OVT, suggesting a role of hostderived immune effectors in OVT. ${ }^{56}$

$\mathrm{x}$. Poxviruses. It is worth noting that NK cells are known to be stressed following surgery, as indicated by their impaired ability to kill target tumor cells and by reduced expression of several activation/maturation markers. This effect has been linked to alterations in levels of signaling cyto/chemokines and suppressor cell populations, and can be reversed by perioperative administration of oncolytic parapoxvirus ovis (ORFV) and vaccinia virus. This reversal was found to correlate with reduced postoperative metastasis formation in mouse melanoma and breast carcinoma models, and was also demonstrated in postoperative cancer surgery patients treated with oncolytic vaccinia virus. These results open new prospects for perioperative therapies using OVs to enhance NK cell function, and thereby reduce metastatic recurrence in cancer surgery patients. ${ }^{57,58}$

\section{Interaction of NK cells with dendritic cells in OVT}

The success of OVT appears to rely on both the innate and adaptive arms of the immune system, and crosstalk between NK and dendritic cells seems important. NK cell-DC interactions have been documented both in vitro and in vivo. Some OVs can boost this response by inducing the immunogenic death of infected tumor cells, leading to DC maturation and probably NK cell activation. The interplay between DCs, NK cells, and tumor cells in OVT has been graphically illustrated in Figure 2. A study focusing on an oncolytic reovirus provides an example of this interplay between infected tumor cells, DCs, and NK cells: DCs primed with reovirus-infected human melanoma oncolysates (DC-MelReo cells) were found to activate NK cells more efficiently than reovirus-infected tumor cells, as revealed by contact-dependent enhancement of IFN- $\gamma$ and chemokine production. DC-MelReo cells underwent maturation and induced NK cell cytotoxicity through type I IFN release. ${ }^{59-61}$ In a mouse prostate cancer model, oncolytic therapy was associated with homing of both CD8 T and NK cells to the tumors, and relied on DCs for chemoattractant production, NK cell activation, and presentation of TAAs to tumor-specific CD8(+) T cells. ${ }^{44}$ The NK-cell-activating capacity of DCs can also be stimulated by direct infection of the latter cells with some OVs. The novel oncolytic virus Maraba MG1 has recently been shown to activate NK cells via direct infection and maturation of DC cells, both cell types being required for MG1-induced reduction of postoperative metastatic disease. ${ }^{62}$ Similar cooperation between DCs and NK cells has been evidenced for recombinant Sendai virus $(\mathrm{rSeV})$. In a murine model, DCs activated through direct $\mathrm{rSeV}$ infection effectively prevented the occurrence of lung metastases. While NK and $\mathrm{CD}^{+}$cells were both found to be crucial to this protection, NK cell activation was not required, suggesting that some DC-dependent immune responses might require NK cells acting as regulators rather than effectors. ${ }^{63}$ 


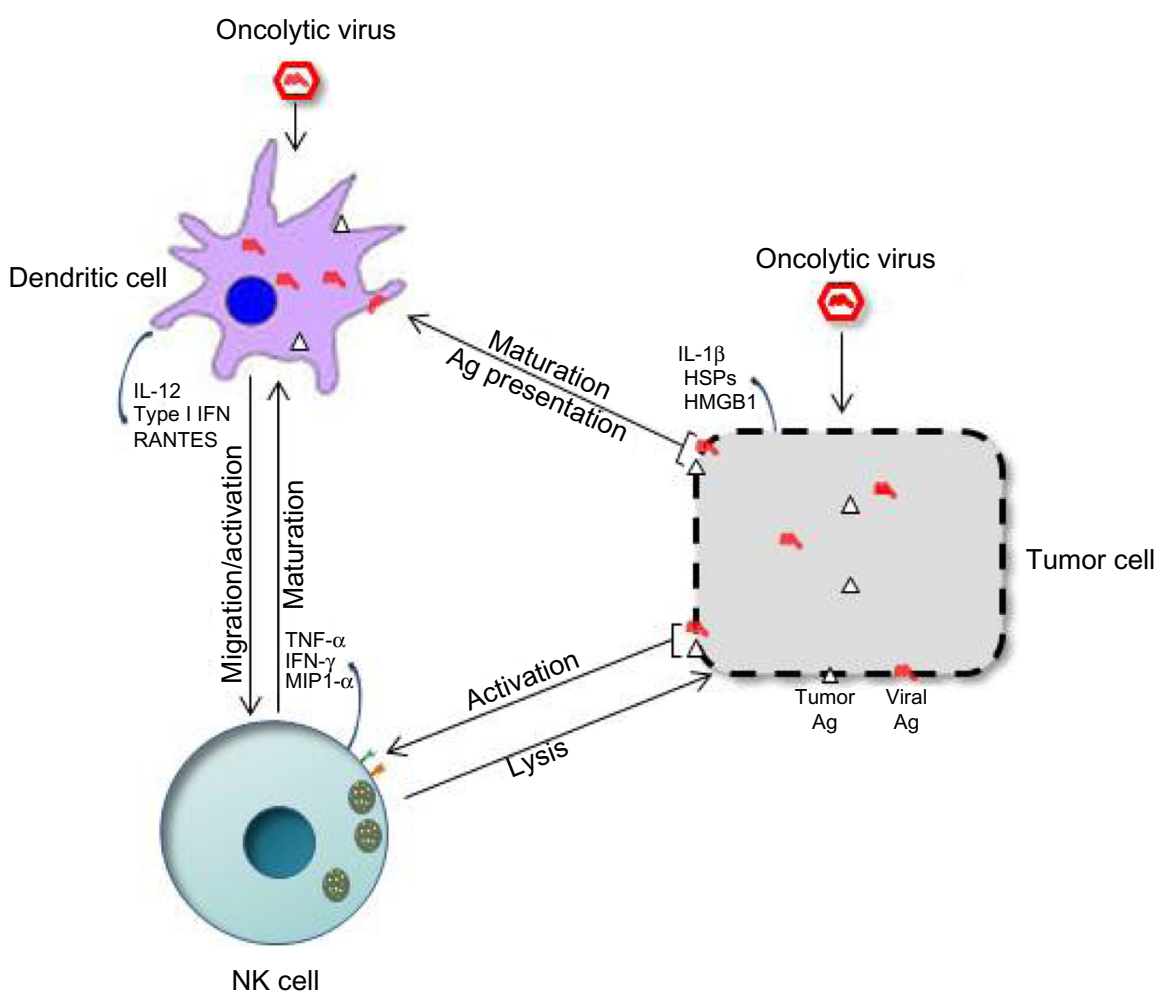

Figure 2 Crosstalk between natural killer (NK) cells, dendritic cells (DCs), and tumor cells in oncolytic virotherapy (OVT).

Notes: Upon infection with oncolytic viruses (OVs), tumor cells produce viral and/or cellular ligands, triggering NK-cell-mediated cytotoxicity and secretion of cytokines, promoting DC maturation and tumor necrosis. In addition, OV infection and/or contact with OV-induced oncolysates triggers DC activation and antigen presentation, which can in turn lead to NK-cell activation through the secretion of cytokines.

\section{NK cells in combinatorial OVT}

\section{Pharmacological modulators}

OVs alone often fail to achieve tumor eradication. To augment the efficacy of OVT, OVs have been engineered to carry cytokine-encoding genes and cause production of immunostimulatory factors, and have also been combined with various pharmacologic modulators. Combination therapy has led to both positive and negative outcomes. In a model of malignant mesothelioma, for example, a combination of cyclophosphamide (CPA) and oVSV proved less effective against tumors than CPA alone. Intratumoral oVSV application caused significant inhibition of both antigen-specific T-cell activation and CPA-activated NK-cell-dependent killing of tumor cells, ${ }^{64}$ in a TGF- $\beta$-dependent manner. In a rat glioma model, intratumoral administration of HSV was associated with a rapid increase in natural killer cells and microglia/macrophages and in IFN- $\gamma$ production. Pretreatment with CPA enhanced HSV replication and direct oncolysis but scaled down the HSV-mediated increase in immune cells and intratumoral IFN- $\gamma .{ }^{65}$ Combination viro/ chemotherapy with the topoisomerase inhibitor irinotecan (CPT-11) and a Sindbis-virus-based vector resulted in longterm survival of about $35 \%$ of tested SCID mice bearing aggressively growing human ovarian tumors, under conditions where single-agent treatments were ineffective. This cooperative therapeutic action was NK-cell-dependent, illustrating the role of these cells in combination treatments with chemotherapeutic drugs. ${ }^{66}$ An immunomodulatory element can also be inserted into the OV backbone. In a xenograft model of lung cancer, inserting $\mathrm{CpG}$ motifs into the adenovirus genome resulted in a significant increase in antitumor efficacy, this improvement being lost in NK-celldepleted animals. ${ }^{67}$

\section{Cytokines and chemokines}

In several cases, arming OVs with transgenes encoding immunomodulatory cytokines has led to successful strengthening of anticancer OVT:

i. IFN- $\beta$ : In a pancreatic cancer model, administration of an adenovirus encoding IFN- $\beta$, in combination with gemcitabine to eliminate suppressor cells, resulted in potent NK-cell-mediated antitumor responses. ${ }^{68}$

ii. IL-2: IL-2 was found to reinforce the effect of Treg depletion on OVT with VSV by increasing virus spread and causing the appearance of activated NK cells possessing enhanced antitumor activity. ${ }^{69}$ 
iii. RANTES: An oncolytic adenovirus expressing the chemokine RANTES has been shown to have an enhanced capacity to recruit DCs, macrophages, NK cells, and $\mathrm{CD}^{+} \mathrm{T}$ cells to the tumor site, triggering Agspecific cytotoxic $\mathrm{T}$ lymphocyte and NK cell responses, thereby causing tumor regression. ${ }^{70}$

iv. HSP70: In a Phase I clinical trial on patients with advanced solid tumors, intratumoral injection of H103, a recombinant oncolytic type 2 adenovirus overexpressing the heat shock protein 70 , caused the number of $\mathrm{T}$ and NK cells to increase. ${ }^{71}$

v. IL-12/IL-18: In a murine melanoma model, intratumoral administration of an oncolytic adenovirus coexpressing cytokines IL-12 and IL-18 led to the infiltration of NK and both $\mathrm{CD}^{+}$and $\mathrm{CD}^{+} \mathrm{T}$ cells into the treated tumor tissues, to better antitumor effects, and to increased survival. $^{72}$

In another study, the efficacy of Sindbis-virus-based vectors against human ovarian carcinoma xenografts was found to be largely NK-cell-dependent and enhanced by IL-12 arming. The enhanced therapeutic effect of Sin/ IL-12 was also NK-cell-dependent and operated through an IFN- $\gamma$-dependent mechanism, inducing the activation of peritoneal macrophages. ${ }^{66}$

vi. IL-15: To exploit the NK-cell-promoting activity of this cytokine, the OV (delNS1) was armed with an IL-15encoding transgene (delNS1-IL-15). Supernatants of delNS1-IL-15-infected (but not of delNS1-infected) melanoma cells induced lysis of noninfected tumor cells, mediated by primary human NK cells. ${ }^{73}$ In a murine metastatic colon adenocarcinoma model, arming of oVSV with a transgene encoding a highly secreted version of human IL-15 improved survival through enhancement of both NK cell number and antitumor T-cell responses. ${ }^{74}$ In another study, oHSVs engineered to express soluble bioactive murine IL-15 alone or in a complex with murine IL-15 receptor $\alpha$ were found to stimulate proliferation of NK cells and to reduce the viability of mouse glioma cells. ${ }^{75}$

vii. GM-CSF: In a murine B16-F10 melanoma model, the oncolytic adenovirus Ad- $\triangle \mathrm{B} 7 / \mathrm{IL} 12 / \mathrm{GMCSF}$ coexpressing IL-12 (targeting NK, T, and NKT cells) and GM-CSF (targeting APCs) promoted antitumor responses and increased survival as compared with a virus expressing IL-12 or GM-CSF alone. In situ delivery of Ad- $\Delta \mathrm{B} 7 / \mathrm{IL} 12 /$ GMCSF resulted in massive infiltration of T cells, CD86 (+) antigen-presenting cells, and NK cells into the tissue surrounding the necrotic area of the tumor. ${ }^{76}$ In a mouse melanoma model, expression of GM-CSF by an engineered oVSV increased its oncosuppressive efficacy, inducing maturation of DCs and NK cells and rapid tumor infiltration by IFN- $\gamma$-producing $\mathrm{T}$ and NK cells. ${ }^{77}$ In a recent study using recombinant oncolytic adenoviruses encoding GM-CSF to treat human cancer patients, the efficiency of OVT was found to correlate with the polymorphisms in gamma receptors that are known to be expressed by innate immune cells. These observations further support the activation and involvement of these innate cells, more particularly NK cells, in OVT using OVs with transgenes encoding immunomodulatory cytokines. ${ }^{78}$

\section{Future strategies and challenges ahead}

OVT has made remarkable progress over the last two decades. Several OVs have been tested for efficacy preclinically and in clinical trials with positive outcomes. There is strong evidence that the immune system plays a critical role in determining the outcome of OVT, and this poses further challenges. An obstacle is host innate immunity, which can impede the success of OVT by clearing the virus from the system. Being important effectors of antiviral defense, NK cells play a dual role in OVT, driving both antitumor and anti-OV responses. Various studies aim to manipulate the functions of NK cells so as to improve the outcome of OVT. As the behavior of NK cells in OVT seems to be both tumor- and OV-dependent, it may be necessary to develop a personalized approach to OVT. To achieve this aim, there is an increasing need to study NK cell responses to OVT in different mouse tumor models (orthotropic, syngeneic) simulating the immunosuppressive microenvironment observed in human tumors. It is essential to develop humanized mouse models where the structural and functional features of immune cells, particularly NK cells, are maintained. Moreover, since combinatorial strategies are likely to be needed, attention must be paid to possible effects of pharmacologic modulators on NK cell subpopulations. Furthermore, viral ligands binding to specific receptors on NK cells should be thoroughly investigated, as blocking of receptor-ligand binding can have important therapeutic implications. In developing future OVT strategies, one should strive to maintain a delicate balance between safety against systemic virus toxicity, oncolysis, and immune-cell-mediated antitumor responses. A more realistic approach might involve the immediate suppression of the innate immune response after viral infection, so as to achieve multiple rounds of viral replication. Once a viral load sufficient for oncolysis is attained, enhancement of antitumor immune effectors, including NK cells, might then contribute to effective tumor clearance. 
Approved or novel (small molecule) drugs may be successively used to achieve the initial dampening of innate and adaptive antiviral defenses to permit robust OV replication followed by the stimulation of antitumor immune response. The success of such a sequential approach is likely to depend on the site and nature of tumor, as well as on the type of oncolytic virus used. Therefore, further preclinical and clinical studies are necessary to identify combinations that maximize the potential of virotherapy through significant synergies. In order to speed up clinical translation, priority should be given to combinations involving chemotherapeutic drugs that are already approved for use in patients. In order to amplify and prolong antitumoral immune response, some of the immunomodulators already discussed in this review may serve to activate immune effector cells, particularly NK cells. It is noteworthy that surgical resection of the tumor represents another complementary treatment to be combined with OVT. However, the implementation of this modality relies on appropriate time intervals being chosen so as to allow OVs to multiply and/ or trigger immune reactions to a sufficient extent before the target tumor is removed.

Irrespective of tumor type, patients receiving OVT appear to respond differently, some benefiting from the treatment more than others. This heterogeneity may be traced back at least in part to variations in the immunocompetence of individual patients. The identification of responsive subjects is therefore crucial to improve the outcome of OVT through the implementation of personalized treatments. Of special interest in this regard is a recent study suggesting that polymorphisms in Fc $\gamma$ receptors displayed in particular by NK cells may play a role in the effectiveness of OVT and serve as predictive and prognostic biomarker for the selection of potentially responsive patients. ${ }^{78}$ Testing and implementing these combinations represent a promising strategy to bring OVs from bench to bedside and to establish OVT as a new effective immunotherapeutic approach.

\section{Acknowledgment}

We thank Dr K Broman for the language editing of our manuscript.

\section{Disclosure}

JR received grants from Oryx $\mathrm{GmbH}$ and Co KG. He also has an ownership interest (patents) in the German Cancer Research Center. Apart from this, the authors declare no conflicts of interest.

\section{References}

1. DePace N. Sulla scomparsa di un enorme vegetante del collo dell'utero senza cura chirugica cancro. La Ginecologia. 1912;9:82-88. Italian.

2. Power A, Bell JC. Cell-based delivery of oncolytic viruses: a new strategic alliance for a biological strike against cancer. Mol Ther. 2007;15(4):660-665.

3. Woller N, Gürlevik E, Ureche CI, Schumacher A, Kühnel F. Oncolytic viruses as anticancer vaccines. Front Oncol. 2014;4:188.

4. Nüesch JP, Rommelaere J. Tumor suppressing properties of rodent parvovirus NS1 proteins and their derivatives. Adv Exp Med Biol. 2014;818:99-124.

5. Nüesch JP, Lacroix J, Marchini A, Rommelaere J. Molecular pathways: rodent parvoviruses - mechanisms of oncolysis and prospects for clinical cancer treatment. Clin Cancer Res. 2012;18(13):3516-3523.

6. Paglino JC, Andres W, van den Pol AN. Autonomous parvoviruses neither stimulate nor are inhibited by the type I interferon response in human normal or cancer cells. J Virol. 2014;88(9):4932-4942.

7. Moehler M, Goepfert K, Heinrich B, et al. Oncolytic virotherapy as emerging immunotherapeutic modality: potential of parvovirus H-1. Front Oncol. 2014;1(4):92.

8. Geletneky K, Huesing J, Rommelaere J, et al. Phase I/IIa study of intratumoral/intracerebral or intravenous/intracerebral administration of Parvovirus H-1 (ParvOryx) in patients with progressive primary or recurrent glioblastoma multiforme: ParvOryx01 protocol. BMC Cancer. 2012;12:99.

9. Cerullo V, Pesonen S, Diaconu I, et al. Oncolytic adenovirus coding for granulocyte macrophage colony-stimulating factor induces antitumoral immunity in cancer patients. Cancer Res. 2010;70(11):4297-4309.

10. Melcher A, Parato K, Rooney CM, Bell JC. Thunder and lightning: immunotherapy and oncolytic viruses collide. Mol Ther. 2011;19(6): 1008-1016.

11. Alvarez-Breckenridge CA, Yu J, Kaur B, Caligiuri MA, Chiocca EA. Deciphering the multifaceted relationship between oncolytic viruses and natural killer cells. Adv Virol. 2012;2012:702839.

12. Marcus A, Gowen BG, Thompson TW, et al. Recognition of tumors by the innate immune system and natural killer cells. Adv Immunol. 2014; 122:91-128.

13. Herberman RB, Nunn ME, Lavrin DH. Natural cytotoxic reactivity of mouse lymphoid cells against syngeneic acid allogeneic tumors. I. Distribution of reactivity and specificity. Int J Cancer. 1975;16:216-229.

14. Kiessling R, Klein E, Wigzell H. "Natural" killer cells in the mouse. I. Cytotoxic cells with specificity for mouse Moloney leukemia cells. Specificity and distribution according to genotype. Eur J Immunol. 1975;5:112-117.

15. Yu J, Freud AG, Caligiuri MA. Location and cellular stages of natural killer cell development. Trends Immunol. 2013;34(12):573-582.

16. Vosshenrich CA, Di Santo JP. Developmental programming of natural killer and innate lymphoid cells. Curr Opin Immunol. 2013;25: $130-138$.

17. Bellora F, Castriconi R, Dondero A, et al. Human NK cells and NK receptors. Immunol Lett. 2014;161(2):168-173.

18. Höglund P, Brodin P. Current perspectives of natural killer cell education by MHC class I molecules. Nat Rev Immunol. 2010;10(10): 724-734.

19. Pegram HJ, Andrews DM, Smyth MJ, Darcy PK, Kershaw MH. Activating and inhibitory receptors of natural killer cells. Immunol Cell Biol. 2011;89(2):216-224.

20. Orr MT, Lanier LL. Natural killer cell education and tolerance. Cell. 2010;142(6):847-856.

21. Ivarsson MA, Michaëlsson J, Fauriat C. Activating killer cell Ig-like receptors in health and disease. Front Immunol. 2014;22(5):184.

22. Ames E, Murphy WJ. Advantages and clinical applications of natural killer cells in cancer immunotherapy. Cancer Immunol Immunother. 2014;63(1):21-28.

23. Liao W, Lin JX, Leonard WJ. Interleukin-2 at the crossroads of effector responses, tolerance, and immunotherapy. Immunity. 2013;38(1): 13-25. 
24. Ochoa MC, Mazzolini G, Hervas-Stubbs S, de Sanmamed MF, Berraondo P, Melero I. Interleukin-15 in gene therapy of cancer. Curr Gene Ther. 2013;13(1):15-30.

25. Ames E, Hallett WH, Murphy WJ. Sensitization of human breast cancer cells to natural killer cell-mediated cytotoxicity by proteasome inhibition. Clin Exp Immunol. 2009;155(3):504-513.

26. Romagné F, André P, Spee P, et al. Preclinical characterization of 1-7F9, a novel human anti-KIR receptor therapeutic antibody that augments natural killer-mediated killing of tumor cells. Blood. 2009;114(13):2667-2677.

27. Welniak LA, Blazar BR, Murphy WJ. Immunobiology of allogeneic hematopoietic stem cell transplantation. Annu Rev Immunol. 2007;25: 139-170.

28. Ruggeri L, Capanni M, Urbani E, et al. Effectiveness of donor natural killer cell alloreactivity in mismatched hematopoietic transplants. Science. 2002;295(5562):2097-2100.

29. Locatelli F, Moretta F, Brescia L, Merli P. Natural killer cells in the treatment of high risk acute leukaemia. Semin Immunol. 2014;26: 173-179.

30. Cheng M, Ma J, Chen Y, et al. Establishment, characterization, and successful adaptive therapy against human tumors of NKG cell, a new human NK cell line. Cell Transplant. 2011;20(11-12):1731-1746.

31. Bhat R, Watzl C. Serial killing of tumor cells by human natural killer cells - enhancement by therapeutic antibodies. PLoS One. 2007;2(3):e326.

32. Jahn T, Zuther M, Friedrichs B, et al. An IL12-IL2-antibody fusion protein targeting Hodgkin's lymphoma cells potentiates activation of NK and T cells for an anti-tumor attack. PLoS One. 2012;7:e44482.

33. Moretta L, Pietra G, Montaldo E, et al. Human NK cells: from surface receptors to the therapy of leukemias and solid tumors. Front Immunol. 2014;5:87.

34. Balsamo M, Manzini C, Pietra G, et al. Hypoxia down regulates the expression of activating receptors involved in NK-cell-mediated target cell killing without affecting ADCC. Eur J Immunol. 2013;43(10): 2756-2764.

35. Altomonte J, Wu L, Chen L, et al. Exponential enhancement of oncolytic vesicular stomatitis virus potency by vextor-mediated suppression of inflammatory responses in vivo. Mol Ther. 2008;16(1):146-153.

36. Altomonte J, Wu L, Meseck M, et al. Enhanced oncolytic potency of vesicular stomatitis virus through vector-mediated inhibition of NK and NKT cells. Cancer Gene Ther. 2009;16(3):266-278.

37. Alvarez-Breckenridge CA, Yu J, Price R, et al. NK cells impede glioblastoma virotherapy through NKp30 and NKp46 natural cytotoxicity receptors. Nat Med. 2012;18(12):1827-1834.

38. Weibel S, Raab V, Yu YA, et al. Viral-mediated oncolysis is the most critical factor in the late-phase of the tumor regression process upon vaccinia virus infection. BMC Cancer. 2011;11:68.

39. Moralès O, Richard A, Martin N, et al. Activation of a helper and not regulatory human $\mathrm{CD} 4+\mathrm{T}$ cell response by oncolytic $\mathrm{H}-1$ parvovirus. PLoS One. 2012;7(2):e32197.

40. Miller CG, Fraser NW. Requirement of an integrated immune response for successful neuroattenuated HSV-1 therapy in an intracranial metastatic melanoma model. Mol Ther. 2003;7(6):741-747.

41. Benencia F, Courrèges MC, Conejo-García JR, et al. HSV oncolytic therapy upregulates interferon-inducible chemokines and recruits immune effector cells in ovarian cancer. Mol Ther. 2005;12(5):789-802.

42. Zhao Q, Zhang W, Ning Z, et al. A novel oncolytic herpes simplex virus type 2 has potent anti-tumor activity. PLoS One. 2014;9(3):e93103.

43. White CL, Twigger KR, Vidal L, et al. Characterization of the adaptive and innate immune response to intravenous oncolytic reovirus (Dearing type 3) during a phase I clinical trial. Gene Ther. 2008;15(12): 911-920.

44. Gujar SA, Pan DA, Marcato P, Garant KA, Lee PW. Oncolytic virusinitiated protective immunity against prostate cancer. Mol Ther. 2011;19(4):797-804.

45. Rintoul JL, Lemay CG, Tai LH, et al. ORFV: a novel oncolytic and immune stimulating parapoxvirus therapeutic. Mol Ther. 2012;20(6): $1148-1157$.
46. Miyamoto $\mathrm{S}$, Inoue $\mathrm{H}$, Nakamura $\mathrm{T}$, et al. Coxsackievirus B3 is an oncolytic virus with immunostimulatory properties that is active against lung adenocarcinoma. Cancer Res. 2012;72(10):2609-2621.

47. Allen C, Vongpunsawad S, Nakamura T, et al. Retargeted oncolytic measles strains entering via the EGFRvIII receptor maintain significant antitumor activity against gliomas with increased tumor specificity. Cancer Res. 2006;66(24):11840-11850.

48. Bhat R, Dempe S, Dinsart C, Rommelaere J. Enhancement of NK cell anti-tumor responses using an oncolytic parvovirus. Int J Cancer. 2011;128(4):908-919.

49. Bhat R, Rommelaere J. NK-cell-dependent killing of colon carcinoma cells is mediated by natural cytotoxicity receptors (NCRs) and stimulated by parvovirus infection of target cells. BMC Cancer. 2013;13:367.

50. Dempe S, Lavie M, Struyf S, et al. Antitumoral activity of parvovirusmediated IL-2 and MCP-3/CCL7 delivery into human pancreatic cancer: implication of leucocyte recruitment. Cancer Immunol Immunother. 2012;61(11):2113-2123.

51. Ogbomo H, Zemp FJ, Lun X, et al. Myxoma virus infection promotes NK lysis of malignant gliomas in vitro and in vivo. PLoS One. 2013;8(6):e66825.

52. Jarahian M, Watzl C, Fournier P, et al. Activation of natural killer cells by new castle disease virus hemagglutinin-neuraminidase. J Virol. 2009;83(16):810-821.

53. Ogbomo H, Michaelis M, Geiler J, et al. Tumor cells infected with oncolytic influenza A virus prime natural killer cells for lysis of resistant tumor cells. Med Microbiol Immunol. 2010;199(2):93-101.

54. Wongthida P, Diaz RM, Galivo F, et al. Type III IFN interleukin-28 mediates the antitumor efficacy of oncolytic virus VSV in immune-competent mouse models of cancer. Cancer Res. 2010;70(11):4539-4549.

55. Heiber JF, Barber GN. Vesicular stomatitis virus expressing tumor suppressor p53 is a highly attenuated, potent oncolytic agent. J Virol. 2011;85(20):10440-10450.

56. Diaz RM, Galivo F, Kottke T, et al. Oncolytic immunovirotherapy for melanoma using vesicular stomatitis virus. Cancer Res. 2007;67(24): 2840-2848.

57. Tai LH, de Souza CT, Bélanger S, et al. Preventing postoperative metastatic disease by inhibiting surgery-induced dysfunction in natural killer cells. Cancer Res. 2013;73(1):97-107.

58. Tai LH, Auer R. Attacking postoperative metastases using perioperative oncolytic viruses and viral vaccines. Front Oncol. 2014;4:217.

59. Prestwich RJ, Errington F, Diaz RM, et al. The case of oncolytic viruses versus the immune system: waiting on the judgment of Solomon. Hum Gene Ther. 2009;20(10):1119-1132.

60. Prestwich RJ, Errington F, Steele LP, et al. Reciprocal human dendritic cell-natural killer cell interactions induce antitumor activity following tumor cell infection by oncolytic reovirus. J Immunol. 2009;183(7):4312-4321.

61. Errington F, Steele L, Prestwich R, et al. Reovirus activates human dendritic cells to promote innate antitumor immunity. J Immunol. 2008;180(9):6018-6026.

62. Zhang J, Tai LH, Ilkow CS, et al. Maraba MG1 virus enhances natural killer cell function via conventional dendritic cells to reduce postoperative metastatic disease. Mol Ther. 2014;22(7):1320-1332.

63. Komaru A, Ueda Y, Furuya A, et al. Sustained and NK/CD4+ T celldependent efficient prevention of lung metastasis induced by dendritic cells harboring recombinant Sendai virus. J Immunol. 2009;183(7): 4211-4219.

64. Willmon C, Diaz RM, Wongthida P, et al. Vesicular stomatitis virusinduced immune suppressor cells generate antagonism between intratumoral oncolytic virus and cyclophosphamide. Mol Ther. 2011;19(1):140-149.

65. Fulci G, Breymann L, Gianni D, et al. Cyclophosphamide enhances glioma virotherapy by inhibiting innate immune responses. Proc Natl Acad Sci U S A. 2006;103(34):12873-12878.

66. Granot T, Venticinque L, Tseng JC, Meruelo D. Activation of cytotoxic and regulatory functions of NK cells by Sindbis viral vectors. PLoS One. 2011;6(6):e20598. 
67. Cerullo V, Diaconu I, Romano V, et al. An oncolytic adenovirus enhanced for toll-like receptor 9 stimulation increases antitumor immune responses and tumor clearance. Mol Ther. 2012;20(11):2076-2086.

68. Suzuki E, Kapoor V, Jassar AS, Kaiser LR, Albelda SM. Gemcitabine selectively eliminates splenic Gr-1+/CD11b+myeloid suppressor cells in tumor-bearing animals and enhances antitumor immune reactivity. Clin Cancer Res. 2005;11(18):6713-6721.

69. Kottke T, Galivo F, Wongthida P, et al. Treg depletion-enhanced IL-2 treatment facilitates therapy of established tumors using systemically delivered oncolytic virus. Mol Ther. 2008;16(7):1217-1226.

70. Lapteva N, Aldrich M, Weksberg D, et al. Targeting the intratumoral dendritic cells by the oncolytic adenoviral vaccine expressing RANTES elicits potent antitumor immunity. J Immunother. 2009;32(2): 145-156.

71. Li JL, Liu HL, Zhang XR, et al. A phase I trial of intratumoral administration of recombinant oncolytic adenovirus overexpressing HSP70 in advanced solid tumor patients. Gene Ther. 2009;16(3):376-382.

72. Choi IK, Lee JS, Zhang SN, et al. Oncolytic adenovirus co-expressing IL-12 and IL-18 improves tumor-specific immunity via differentiation of T cells expressing IL-12R $\beta 2$ or IL-18R $\alpha$. Gene Ther. 2011;18(9): 898-909.
73. van Rikxoort M, Michaelis M, Wolschek M, et al. Oncolytic effects of a novel influenza A virus expressing interleukin-15 from the NS reading frame. PLoS One. 2012;7(5):e36506.

74. Stephenson KB, Barra NG, Davies E, Ashkar AA, Lichty BD. Expressing human interleukin-15 from oncolytic vesicular stomatitis virus improves survival in a murine metastatic colon adenocarcinoma model through the enhancement of anti-tumor immunity. Cancer Gene Ther. 2012;19(4):238-246.

75. Gaston DC, Odom CI, Li L, et al. Production of bioactive soluble interleukin-15 in complex with interleukin-15 receptor alpha from a conditionally-replicating oncolytic HSV-1. PLoS One. 2013;8(11):e81768.

76. Choi KJ, Zhang SN, Choi IK, Kim JS, Yun CO. Strengthening of antitumor immune memory and prevention of thymic atrophy mediated by adenovirus expressing IL-12 and GM-CSF. Gene Ther. 2012;19(7): 711-723.

77. Lemay CG, Rintoul JL, Kus A, et al. Harnessing oncolytic virusmediated antitumor immunity in an infected cell vaccine. Mol Ther 2012;20(9):1791-1799.

78. Hirvinen M, Heiskanen R, Oksanen M, et al. Fc-gamma receptor polymorphisms as predictive and prognostic factors in patients receiving oncolytic adenovirus treatment. J Transl Med. 2013;11:193.

\section{Publish your work in this journal}

ImmunoTargets and Therapy is an international, peer-reviewed open access journa focusing on the immunological basis of diseases, potential targets for immune based therapy and treatment protocols employed to improve patient management Basic immunology and physiology of the immune system in health, and disease will be also covered. In addition, the journal will focus on the impact of manage-

\section{Dovepress}

ment programs and new therapeutic agents and protocols on patient perspectives such as quality of life, adherence and satisfaction. The manuscript management system is completely online and includes a very quick and fair peer-review system, which is all easy to use. Visit http://www.dovepress.com/testimonials.php to read real quotes from published authors.

Submit your manuscript here: http://www.dovepress.com/immunotargets-and-therapy-journal 